\title{
Generalized Hopfian Property, a Minimal Haken Manifold, and Epimorphisms Between 3-Manifold Groups
}

\author{
REID Alan W. \\ Department of Mathematics, University of Texas, Austin, TX 78712, USA \\ E-mail: areid@antfire.ma.utexa.edu \\ WANG Shi Cheng \\ Department of Mathematics, Peking University, Beijing 100871, P. R. China \\ E-mail: swang@sxx0.math.pku.edu.cn \\ ZHOU Qing \\ Department of Mathematics, East China Normal University, Shanghai 200062, P. R. China \\ E-mail: qzhou@math.ecnu.edu.cn
}

\begin{abstract}
We address the question that if $\pi_{1}$-surjective maps between closed aspherical 3-manifolds have the same rank on $\pi_{1}$ they must be of non-zero degree. The positive answer is proved for Seifert manifolds, which is used in constructing the first known example of minimal Haken manifold. Another motivation is to study epimorphisms of 3-manifold groups via maps of non-zero degree between 3manifolds. Many examples are given.
\end{abstract}

Keywords Non-zero degree maps, Epimorphisms, 3-Manifolds

2000 MR Subject Classification 55N05, 57M05, 57N10

\section{Introduction and Some Examples}

Let $M$ and $N$ be closed 3-manifolds and $f: M \rightarrow N$ a map of non-zero degree. Then the image of $f_{*}$ is a subgroup of finite index in $\pi_{1}(N)$. If $M$ and $N$ are aspherical, any homomorphism $\phi: \pi_{1}(M) \rightarrow \pi_{1}(N)$ determines a unique map $f: M \rightarrow N$ up to homotopy such that $f_{*}=\phi$. It seems natural to ask does there exist $f: M \rightarrow N$ of non-zero degree, given a homomorphism $\phi$ surjecting $\pi_{1}(M)$ onto a subgroup of finite index in $\pi_{1}(N)$ ?

Received September 16, 1999, Revised August 31, 2000, Accepted March 29, 2001.

We would like to thank RONG Yongwu and the referee for many helpful comments. The first author was supported by the Royal Society, the NSF and The Alfred P. Sloan Foundation. The second and third authors are supported by MSTC, by Quisi and by Outstanding Youth Fellowship of NSFC. 
There are elementary constructions of examples (see below) that show, in general, that the answer is no. Before discussing some examples we make the following definition:

Definition 1.1 A map $f: M \rightarrow N$ between 3-manifolds is $\pi_{1}$-surjective (resp. $\pi_{1}$-finiteindex) if $f_{*}: \pi_{1}(M) \rightarrow \pi_{1}(N)$ is surjective (resp. the image of $f_{*}$ is a subgroup of finite index).

Recall that if $M$ is an $n$-manifold, the rank of $\pi_{1}(M)$ (or just $M$ by abuse language) is the minimal cardinality of a generating system for $\pi_{1}(M)$.

We first consider the situation in dimension 2, which is quite simple. Throughout the paper $\Sigma_{k}$ will denote a closed orientable surface of genus $k$.

Example 1.1 It is not difficult to see that there is a $\pi_{1}$-surjective map $f: \Sigma_{l} \rightarrow \Sigma_{k}$ which is of degree zero when $l \geq 2 k$.

On the other hand, if $f: \Sigma_{l} \rightarrow \Sigma_{k}$ is a $\pi_{1}$-surjective map with $0<l<2 k$, then we claim that $f$ is of non-zero degree. To see this, choose a 1-skeleton of $\Sigma_{k}$ to be a one point wedge of $2 k$ circles $\mathcal{V}=\vee C_{i}$. Fix a point $x_{i}$ on $C_{i}$. If $f$ is of degree zero, then the image of $f$ can be deformed into $\mathcal{V}$. We assume therefore that this is the case. Since $f_{*}$ is $\pi_{1}$-surjective, $f: \Sigma_{l} \rightarrow \mathcal{V}$ must be surjective. We may also assume that $f$ is transversal to each $x_{i}, i=1,2, \ldots, 2 k$. So $f^{-1}\left(\cup x_{i}\right)$ is a set of essential circles. Partition $f^{-1}\left(\cup x_{i}\right)$ into sets $G_{1}, \ldots, G_{h}$ such that two components are in the same set if and only if they are parallel. For each $G_{j}$, find an annulus $A_{j}$ containing $G_{j}$. Then squeeze each $A_{j}$ to an arc $a_{j}$ and the part $\Sigma_{k} \backslash \cup A_{j}$ to a point. The quotient $Q$ will be a bouquet of $h$ circles. Since $\mathcal{V}-\left\{x_{i}, i=1,2, \ldots, 2 k\right\}$ is contractible, the map $f: \Sigma_{l} \rightarrow \mathcal{V}$ factors through $q: Q \rightarrow \mathcal{V}$ which is still $\pi_{1}$-surjective. It follows that $h \geq 2 k$. In particular, there are at least $h \geq 2 k$ disjoint essential non-separating non-parallel circles. By a well-known argument in surface topology, we must have that $l$, the genus of $\Sigma_{l}$, is at least $2 k$.

Returning to dimension 3, the first example illustrates the aspherical assumption.

Example 1.2 Let $f=e \circ p: S^{2} \times S^{1} \rightarrow S^{2} \times S^{1}$, where $p$ is a map which pinches $S^{2} \times S^{1}$ to $S^{1}$, and $e$ identifies $S^{1}$ to a fiber $* \times S^{1} \subset S^{2} \times S^{1}$. Clearly $f$ is of zero degree but $\pi_{1}$-surjective.

Example 1.3 shows that if we do not require that the manifolds have the same rank, then the answer to the question is no.

Example 1.3 We construct a map $f: \Sigma_{g+1} \times S^{1} \rightarrow \Sigma_{g} \times S^{1}$ of zero degree which is $\pi_{1^{-}}$ surjective. The map $f$ is the composition of the following four geometric operations:

Project $\Sigma_{g+1} \times S^{1}$ to $\Sigma_{g+1}$;

Squeeze a suitable separating circle on $\Sigma_{g+1}$ to a point in such a way that the quotient space is a one point union of a torus and $\Sigma_{g}$;

Squeeze the torus to a circle in such a way that the quotient space is a one point union of the circle and $\Sigma_{g}$;

Send $\Sigma_{g}$ and the circle to a section $\Sigma_{g} \times *$ and the circle fiber of $\Sigma_{g} \times S^{1}$ respectively.

Example 1.4 has the same purpose as Example 1.3, but the manifolds in this case are 
hyperbolic.

Example 1.4 Let $M$ be a closed hyperbolic 3-manifold whose fundamental group surjects the free group of rank 2. Such examples are easily constructed by doing hyperbolic surgery on a null-homotopic hyperbolic knot in $\Sigma_{2} \times S^{1}$ [Section 3,1 ]. Let $\phi_{1}: \pi_{1}(M) \rightarrow \mathbb{Z} * \mathbb{Z}$ denote such a map. Let $N$ be any hyperbolic 3 -manifold such that $\pi_{1}(N)$ has two generators. Then there is an epimorphism $\phi_{2}: \mathbb{Z} * \mathbb{Z} \rightarrow \pi_{1}(N)$. If we choose $N$ such that the volume of $N$ is larger than the volume of $M$, then the map realizing the epimorphism $\phi=\phi_{2} \circ \phi_{1}$ must be of zero degree, by the work of Gromov and Thurston. We remark that the volumes of hyperbolic 3manifolds of rank 2 are unbounded. Briefly, it follows from the work of Adams that the volumes of hyperbolic 2-bridge knot complements are unbounded. Doing large enough hyperbolic Dehn surgeries on these gives the required family, see [2].

In fact it can be seen directly that the map realizing $\phi$ must be of zero degree since such a map factors through a 1-dimensional complex.

As a consequence of these examples, we state the following more refined version of the question posed above:

Question 1.5 Let $M$ and $N$ be closed aspherical 3-manifolds such that the rank of $\pi_{1}(M)$ equals the rank of $\pi_{1}(N)$. Assume that $\phi: \pi_{1}(M) \rightarrow \pi_{1}(N)$ is surjective or its image is a subgroup of finite index. Does $\phi$ determine a map $f: M \rightarrow N$ of non-zero degree?

Note that if $M$ and $N$ are homeomorphic and satisfy Thurston's geometrization conjecture, then a $\pi_{1}$-surjective map $f: M \rightarrow M$ must be of degree one. For since $\pi_{1}(M)$ is Hopfian, that $f_{*}$ is surjective implies $f_{*}$ is an isomorphism. Since $M$ is aspherical $f$ must be a homotopy equivalence, and so, in particular, $f$ is of degree one. Thus the question above is a kind of generalization of the Hopfian property: the condition "homeomorphic manifolds" is replaced by "manifolds of the same rank", the condition " $\pi_{1}$-surjective" is replaced by " $\pi_{1}$-surjective" or " $\pi_{1}$-finite-index", and in conclusion replace "degree one" by "non-zero degree". It is easy to construct examples to show that "non-zero degree" cannot be sharpened to "degree one", see the examples in Section 3.

One of the main results of this paper is to prove that Question 1.5 has a positive answer for Seifert fibered 3-manifolds (see Theorem 2.1 and Remark 2.4).

In Section 4 we use this result to construct the first known example of a Haken 3-manifold which is minimal with respect to degree 1 mappings in Thurston's picture of 3 -manifolds (Theorem 4.1). The manifold is a graph manifold built from the union of two trefoil knot complements. An orientable 3-manifold $M$ is minimal if given a degree-one map $f: M \rightarrow N$ implies either $N=S^{3}$ or $M=N$. Usually it is difficult to tell if a 3 -manifold is minimal. We remark that all minimal Seifert manifolds are non-Haken and that the known minimal hyperbolic 3-manifolds are also non-Haken, see [1], [3] and [4] for a further discussion of such matters.

We are also motivated by the following:

[5, Problem 1.12 (J. Simon)] Let $G_{K}=\pi_{1}\left(S^{3}-K\right)$ for a knot $K$ in $S^{3}$. Conjectures: If there is a non-trivial epimorphism $\phi: G_{K} \rightarrow G_{L}$, then 
(A) rank $G_{L}>\operatorname{rank} G_{K}$;

(B) $\operatorname{genus}(L) \geq \operatorname{genus}(K)$;

(C) Given $K$, there is a number $N_{K}$ such that any sequence of epimorphisms of knot groups $G_{K} \rightarrow G_{L_{1}} \rightarrow \ldots . \rightarrow G_{L_{n}}$ with $n \geq N_{K}$ contains an isomorphism;

(D) Given $K$, there are only finitely many knot groups $G$ for which there is an epimorphism $G_{K} \rightarrow G$.

These conjectures have seen little progress. On the other hand, more recently, questions similar to (C) and (D) have been raised for degree-one maps and there are already several substantial results in this setting.

[5, Problem 3.100 (Y. Rong)] Let $M$ be a closed orientable 3-manifold.

(A) Are there only finitely many irreducible 3 -manifolds $N$ such that there exists a degreeone map $M \rightarrow N$ ?

(B) Does there exist an integer $N_{M}$ such that given a sequence of degree-one maps $M \rightarrow$ $M_{1} \rightarrow \ldots . \rightarrow M_{k}$ with $k>N_{M_{0}}$, the sequence contains an homotopy equivalence?

If one assumes Thurston's geometrization conjectural picture of 3-manifolds, the answer to (B) is yes if $k=\infty$ [6] by Rong, or each $M_{i}$ is Haken [7] by Soma; the answer to (A) is yes if the targets have one of Thurston's eight geometries: for the hyperbolic case [8] by Soma, for the spherical case [9] by Hayat-Legrand-Wang-Zieschang and the remaining cases [10] by Wang-Zhou.

Thus it seems natural to study the conjectures of J. Simon for closed orientable 3-manifolds (Question 3.1 in Section 3). We find that the positive answer for Question 1.5 is important for studying the conjectures. This will be addressed in Section 3 .

\section{$2 \quad \pi_{1}$-Surjective Maps Between Aspherical Seifert Manifolds}

Theorem 2.1 Let $M_{1}$ and $M_{2}$ be closed orientable aspherical Seifert fiber spaces with the same rank their base orbifolds be orientable. Then any $\pi_{1}$-surjective map $f: M_{1} \rightarrow M_{2}$ is of non-zero degree.

To prove Theorem 2.1, we will make use of Rong [11]; in particular, we refer the reader to [11] for the definition of a vertical pinch, a squeeze, a squeeze torus, and vertical squeeze. Suppose $T$ is a vertical torus in a Seifert manifold $M$ with induced $S^{1}$ fibration. Let $C$ be a circle on $T$ which meets each $S^{1}$ fiber in exactly one point. Then $T$ can be parameterized as $C \times S^{1}$. Call the quotient map $C \times S^{1} \rightarrow S^{1}$ by squeezing $C \times *$ to $*$ for each $* \in S^{1}$ a standard squeeze.

Also recall that any orientable Seifert manifold $M$ with orientable base orbifold of genus $g$ and with $n$ singular fibers has a unique normal form $\left(g ; b ; \alpha_{1}, \beta_{1} ; \ldots ; \alpha_{k}, \beta_{k}\right)$, where $0 \leq \beta_{i} \leq \alpha_{i}$, $i=1, \ldots, k$. The orbifold $O_{1}$ of $M_{1}$ will be denoted by $\left(g ; \alpha_{1}, \ldots, \alpha_{k}\right)$. In the case when $g=0$, we usually omit the reference to $g$. 
In preparation for the proof of Theorem 2.1, we begin with two lemmas.

Lemma 2.2 Let $f: M \rightarrow N$ be a map between aspherical Seifert manifolds and $1 \neq f_{*}(h) \subset$ $h^{\prime}$, where $M$ is closed and $\partial N \neq \emptyset, h$ and $h^{\prime}$ are regular fibers of $M$ and $N$, respectively. Then either $f$ admits a standard squeeze along an incompressible vertical torus, or $f$ can be homotoped so that the image of $f$ lies in a fiber of $N$.

Proof Lemma 2.2 is exactly Lemma 3.5 of [11] but with "standard squeeze" being replaced by "vertical squeeze". However, one can verify that in Case (2) of the proof of Lemma 3.5 of [11], the vertical squeeze there is indeed a standard squeeze. Since for a fiber preserving map $p$ from an $S^{1}$-fibered torus $T$ to an $S^{1}$ fibered annulus $A$, the kernel of $p$ and the $S^{1}$-fiber generate the $\pi_{1}(T)=\mathbb{Z} \oplus \mathbb{Z}$, therefore the circle on $T$ generating the kernel must intersect the fiber in exactly one point.

Lemma 2.3 Suppose $f: F \rightarrow O$ is an orbifold branch covering, where $F$ is a surface of genus $g$, and $O$ is a orbifold, both being orientable and having non-positive Euler characteristics. Then $\operatorname{rank}\left(\pi_{1}(F)\right) \geq \operatorname{rank}\left(\pi_{1}(O)\right)-1$ if $f$ is a double-branched cover over 2 -sphere, and $\operatorname{rank}\left(\pi_{1}(F)\right) \geq$ $\operatorname{rank}\left(\pi_{1}(O)\right)$ if otherwise.

Proof The proof is based on the results about the ranks of Fuchsian groups [12, Theorem 4.16.1] and the Riemann-Hurwitz formula.

Suppose $O$ has $k$ singular points of index $v_{i}, i=1, \ldots, k$, with the underlying space of genus $g^{\prime}$ and the degree of $f$ is $n$. Then we have

$$
2-2 g=n\left(2-2 g^{\prime}-\sum_{i=1}^{k}\left(1-\frac{1}{v_{i}}\right)\right) .
$$

For the case $g=1$, the verification is direct, so we assume below that $g>1$.

If $n=2$ then all $v_{i}=2, k=2 m$ and we have $2-2 g=2\left(2-2 g^{\prime}-m\right)$, i.e., $g=2 g^{\prime}+m-1$. Now $\operatorname{rank}\left(\pi_{1}(F)\right)=2 g=4 g^{\prime}+2 m-2$ and the $\operatorname{rank}\left(\pi_{1}(O)\right)$ is at most $2 g^{\prime}+2 m-1$ if $g^{\prime}>0$ and is $2 m-1$ if $g^{\prime}=0$ by [12, Theorem 4.16.1]. In any case, the lemma follows.

If $n \geq 3$, then

$$
2-2 g \leq 3\left(2-2 g^{\prime}-\sum_{i=1}^{k}\left(1-\frac{1}{v_{i}}\right)\right) \leq 3\left(2-2 g^{\prime}-\frac{k}{2}\right),
$$

i.e., $2 g \geq 6 g^{\prime}-4+\frac{3 k}{2}$. If $g^{\prime}>0,2 g \geq 2 g^{\prime}+\frac{3 k}{2}$. But the rank of $\pi_{1}(O)$ is, at most, $2 g^{\prime}+k-1$. If $g^{\prime}=0$, then we have $2 g \geq-4+\frac{3 k}{2}$ if $k$ is even and $g \geq-4+\frac{3 k}{2}+\frac{1}{2}$ if $k$ is odd. The rank of $\pi_{1}(O)$ is, at most, $k-1$. It follows that if $k \leq 5$, then $2 g \geq k-1$. If $k \leq 4$ we still have $2 g \geq k-1$ since we assume that $g>1$.

Proof of Theorem 2.1 Suppose $f$ is of zero degree. For clarity, the proof is divided into three steps.

Step (1) We prove the following

Claim: $\quad f(h)$ is homotopically non-trivial, where $h$ is the regular fiber of $M_{1}$. 
Proof of Claim Let $M_{1}=\left(g ; b ; a_{1}, b_{1} ; \ldots ; a_{k}, b_{k}\right)$ and $G_{1}=\pi_{1}\left(M_{1}\right) /\langle h\rangle$, where $\langle h\rangle$ is the cyclic group generated by the regular fiber of $M_{1}$. Let $r=\operatorname{rank}\left(\pi_{1}\left(M_{1}\right)\right)=\operatorname{rank}\left(\pi_{2}\left(M_{2}\right)\right)$.

By [13, Theorem 1.1] and [12, Theorem 4.16.1], one of the following cases holds:

(1) $\operatorname{rank}\left(\pi_{1}\left(M_{1}\right)\right)>\operatorname{rank}\left(G_{1}\right)$;

(2) $\operatorname{rank}\left(\pi_{1}\left(M_{1}\right)\right)=\operatorname{rank}\left(G_{1}\right)$; there is a set of generators of $G_{1}$ which realizes the rank and contains at least one torsion element;

(3) $\operatorname{rank}\left(\pi_{1}\left(M_{1}\right)\right)=\operatorname{rank}\left(G_{1}\right)=\operatorname{rank}\left(G_{1} / T\right)$, where $T$ is the normal subgroup normally generated by the torsion elements and $G_{1} / T$ is a surface group.

If $f(h)$ is homotopically trivial, then $f_{*}: \pi_{1}\left(M_{1}\right) \rightarrow \pi_{1}\left(M_{2}\right)$ induces an epimorphism $\phi$ : $G_{1} \rightarrow \pi_{1}\left(M_{2}\right)$.

In Case (1), the Claim is clearly true.

In Case (2), the Claim is also true since $\pi_{1}\left(M_{2}\right)$ is torsion free.

In Case (3), $f_{*}$ induces an epimorphism $\phi^{\prime}: G_{1} / T \rightarrow \pi_{1}\left(M_{2}\right)$. Let $f^{\prime}: F \rightarrow M_{2}$ be the map which realizes $\phi^{\prime}$. Since $\phi^{\prime}$ is not injective, (otherwise $\phi^{\prime}$ would be an isomorphism and $\pi_{1}\left(M_{2}\right)$ would be a surface group), by the simple loop theorem for maps from a surface to a Seifert manifold [14], there are essential simple loops in the kernel of $\phi^{\prime}$. Assume first there is an essential non-separating simple loop, which we denote by $\alpha$, in the kernel. Then the map $f^{\prime}$ induces a map $f^{\prime \prime}: F^{\prime} \rightarrow M_{2}$, where $F^{\prime}$ is a complex obtained by squeezing $F$ along $\alpha$. It is easy to see that the rank of $\pi_{1}\left(F^{\prime}\right)$ is $r-1$. We reach a contradiction. If all essential simple loops in the kernel of $\phi^{\prime}$ are separating, let $\alpha$ be a maximal family of non-parallel separating essential simple closed curves in kernel $\phi^{\prime}$. Again $f^{\prime}$ can factor through $f^{\prime \prime}: F^{\prime} \rightarrow M_{2}$, where $F^{\prime}$ is a complex obtained by squeezing $F$ along $\alpha$, which is a union of closed surfaces connected by arcs. Let $S$ be a surface in $F^{\prime}$. Due to the maximality of $\alpha$, the restriction $\left.f^{\prime \prime}\right|_{S}$ is $\pi_{1}$-injective, which must be either horizontal or vertical by [14]. If $\left.f^{\prime \prime}\right|_{S}$ is horizontal, then $p_{2} \circ f^{\prime \prime} \mid: S \rightarrow O\left(M_{2}\right)$ is an orbifold branched covering, where $p_{2}: M_{2} \rightarrow O\left(M_{2}\right)$ is the fiber map. But the rank of $\pi_{1}(S)$ is at most $r-2$. This is also ruled out by Lemma 2.3. If $\left.f^{\prime \prime}\right|_{S}$ is vertical for each surface $S$ of $F^{\prime}$, then $F^{\prime}$ contains, at most, $g$ such surfaces and each of them is a torus. Clearly the rank of $f_{*}^{\prime \prime} \pi_{1}\left(F^{\prime}\right)$ is at most $g+1$, which is at most $r-1$ (since $g>1$ and $r \geq 2 g$ ). Again we reach a contradiction.

Step (2) We will factor $f: M_{1} \rightarrow X \rightarrow M_{2}$, where the 2-dimensional complex $X$ is a quotient of $M$ with rank $r_{X}$.

Since $f(h)$ is homotopically non-trivial and $f_{*}$ is surjective, a standard argument in 3manifold topology shows that $f: M_{1} \rightarrow M_{2}$ can be deformed to be a fiber-preserving map (see [15] for example). Suppose the mapping degree is zero. We can further deform the map so that the image $f\left(M_{1}\right)$ misses a regular fiber $h^{\prime}$ of $M_{2}$. To see this, $f: M_{1} \rightarrow M_{2}$ is fiber preserving. We can further deform $f$ so that for each singular fiber of $M_{2}$, its pre-image consists of finitely many fibers of $M_{1}$. Let $S_{i}$ be the union of singular fibers of $M_{1}$. Now remove $f^{-1}\left(f\left(S_{1}\right) \cup S_{2}\right)$ from $M_{1}$ and remove $f\left(S_{1}\right) \cup S_{2}$ from $M_{2}$. The restriction of $f$ gives a proper map $f^{\prime}: M_{1}^{\prime} \rightarrow M_{2}^{\prime}$, which is a fiber-preserving map between circle bundles. Since $f$ is assumed to be of degree zero, $f^{\prime}$ is of zero degree. Since $f(h)$ is non-trivial, the induced proper map $\bar{f}^{\prime}: F_{1}^{\prime} \rightarrow F_{2}^{\prime}$ between 
base surfaces must be of degree zero. Hence $\bar{f}^{\prime}$ can be deformed so that its image misses a point of $F_{2}^{\prime}$. This deformation can be lifted to the bundle map $f^{\prime}$ whose image then misses a circle fiber in $M_{2}^{\prime}$. With this we reach the situation claimed above.

Now remove an open-fibered neighborhood of $h^{\prime}$, and denote the resulting manifold by $N$. Then we have a fiber-preserving map $f: M_{1} \rightarrow N$, where $\partial N \neq \emptyset$.

According to Lemma 2.2, either $f: M_{1} \rightarrow N$ admits a standard squeeze along an incompressible vertical torus, or $f\left(M_{1}\right) \subset$ a fiber of $N$. Using this we can reformulate the above so that either $f: M_{1} \rightarrow M_{2}$ admits a standard squeeze along an incompressible vertical torus, or $f\left(M_{1}\right) \subset$ a fiber of $M_{2}$.

Since $f$ is $\pi_{1}$-surjective, and $M_{2}$ is an closed aspherical Seifert fiber space, the situation, $f\left(M_{1}\right) \subset$ a fiber of $M_{2}$, cannot happen. Let $\mathcal{T}$ be a maximal family of disjoint non-parallel incompressible tori along which $f$ admits a standard squeeze. Let $X_{1}=\mathcal{Q} \cup \mathcal{A}$ be the space obtained after the squeezing, where $\mathcal{Q}$ is a union of Seifert fiber spaces with the induced Seifert fibration, $\mathcal{A}$ is a union of annuli and $\partial \mathcal{A}$ is a union of regular fibers of $\mathcal{Q}$ (due to a standard squeeze). Then $f$ induces a $\pi_{1}$-surjective map $X_{1} \rightarrow M_{2}$, which we continue to denote by $f$.

Suppose first $g>0$. Then $f$ admits a standard squeeze along a non-separating torus (Indeed by arguments before, we can assume that $f$ is fiber preserving and the image of $f$ misses a regular fiber of $M_{2}$. Then $f$ induces a map $\bar{f}$ from a closed surface $\Sigma_{g}$ to a punctured surface, and it is known that $\bar{f}$ admits a squeeze along a non-separating circle $C$ on $\Sigma_{g}$, which will provide a non-separating squeeze torus of $f$, and then the squeeze can be chosen to be standard by Lemma 2.2). Moreover, if $X_{1}$ is obtained from $M$ by a standard squeeze along a non-separating torus, then the rank of $\pi_{1}\left(X_{1}\right)$ is $r-1$, which will be a contradiction.

Below we assume that $g=0$. Then every squeeze torus is a separating torus. And therefore, each annulus in $\mathcal{A}$ is separating.

Now all components of $\mathcal{Q}$ are Seifert fibered spaces with the induced Seifert fibrations, so we may assume that $Q_{1}, \ldots, Q_{k_{1}}$ are Seifert manifolds of $\mathcal{Q}$ which are not the trivial circle bundle over $S^{2}$ and $Q_{k_{1}+1}, \ldots, Q_{k_{1}+k_{2}}$ are trivial circle bundles over $S^{2}$. Clearly each $Q_{j}, j>k_{1}$, is $S^{2} \times S^{1}$ and which is connected by at least three annuli in $\mathcal{A}$.

Each component $Q_{i}$ of $\mathcal{Q}$ must have an infinite fundamental group, otherwise $f(h)$ is an element of finite order, which must be trivial in $\pi_{1}\left(M_{2}\right)$, and this is forbidden by Step (1). In particular, we have

Fact 1 Each $Q_{i}$ contains at least 2 singular fibers, $i=1, \ldots, k_{1}$.

We will also verify the following

Fact $2 \quad k_{1}+k_{2} \leq k-2$.

Representing each component of $\mathcal{Q}$ by a vertex and each component of $\mathcal{A}$ by an edge, we get a connected tree $G$ of $v=k_{1}+k_{2}$ vertices, and at least $k_{2}$ vertices have valence at least 3 . Then the number of edges $e$ is at least $\frac{1}{2}\left(k_{1}+3 k_{2}\right)$. By the Euler characteristic formula we have $1=v-e \leq k_{1}+k_{2}-\frac{1}{2}\left(k_{1}+3 k_{2}\right)=\frac{k_{1}}{2}-\frac{k_{3}}{2}$, i.e., $k_{2} \leq k_{1}-2$. By Fact 1 , we have $k_{1} \leq k / 2$, therefore $k_{1}+k_{2} \leq k-2$.

By the maximality of $\mathcal{T}$, each $Q_{i}$ contains no squeeze torus for $\left.f\right|_{Q_{i}}$, so we have that $f\left(Q_{i}\right) \subset$ 
a fiber of $M_{2}$, and consequently we have the following

Fact 3 Each $Q_{i}$ has base orbifold $S^{2}$ and has no more than 3 singular fibers (otherwise, there will be a squeeze torus).

So $f: X_{1} \rightarrow M_{2}$ induces a $\pi_{1}$-surjective map $X=\mathcal{S} \cup \mathcal{A}_{1} \rightarrow M_{2}$, where $\mathcal{S}$ is a union of $v=k_{1}+k_{2}$ circles, which are amalgamated by $e$ annuli. Hence $\pi_{1}(X)$ has a presentation of $k_{1}+k_{2}$ generators with $e$ relations. Therefore its rank $r_{X}$ is at most $k_{1}+k_{2}$.

Step (3) We will show that $r_{X}<r$ and then reach a contradiction.

Say that $M_{1}$ is of type I, if $M_{1}$ has normal form $\left(0 ; b ; 2,1 ; \ldots ; 2,1 ; 2 \lambda+1, b_{k}\right)$, where $k \geq 4$ is even, and $\lambda>1$, otherwise call $M_{1}$ of type II. By [13, Theorem 1.1], then $r=k-2$ if $M_{1}$ is of type I and $r=k-1$ if $M_{1}$ is of type II.

Moreover, if $M_{1}$ is of type $\mathrm{I}$, then at least one $Q_{i}$ contains 4 singular fibers (since $\lambda>1$ and both $\left(0 ; b ; 2,1 ; 2 \lambda+1, b_{2}\right)$ and $\left(0 ; b ; 2,1 ; 2,1 ; 2 \lambda+1, b_{3}\right)$ have finite fundamental groups), which is not possible by Fact 3 .

If $M_{1}$ is of type II, then $r=k-1$ and $k \geq 3$, but

$$
r_{X} \leq k_{1}+k_{2} \leq k-2<k-1=r
$$

Corollary 2.4 Suppose $M_{1}$ and $M_{2}$ are closed orientable Seifert fiber spaces with infinite fundamental groups and the orbifold of $M_{1}$ has the underlying space $S^{2}$ and $\operatorname{rank}\left(\pi_{1}\left(M_{1}\right)\right)<2$ $\operatorname{rank}\left(\pi_{1}\left(M_{2}\right)\right)$. If a map $f: M_{1} \rightarrow M_{2}$ is $\pi_{1}$-surjective, then $f$ is of non-zero degree.

Proof Now $\pi_{1}\left(O_{1}\right)$ is generated by torsion elements. So Step 1 in the proof of Theorem 2.1 is passed.

Let $r, k, r_{X}$ be defined as in the proof of Theorem 2.1, and $r_{2}$ be the rank of $\pi_{1}\left(M_{2}\right)$. If $f: M_{1} \rightarrow M_{2}$ is $\pi_{1}$-surjective map and is of zero degree, then $r+1=k \geq 2 r_{X} \geq 2 r_{2}$ by the end of the proof of Theorem 2.1.

Remark In Theorem 2.1, the condition " $f$ is $\pi_{1}$-surjective" can be replaced by " $f$ is $\pi_{1}$ finite-index", and the condition "orbifolds are orientable" can be removed. For details see [16], where the proof is parallel to the proof above, but involves a more complicated case by case argument.

Also the proof in [16] needs the following

Proposition 2.5 Let $M_{1}$ and $M_{2}$ be closed orientable aspherical Seifert fiber spaces with orientable base orbifolds of the same genus $g$. Suppose there is a $\pi_{1}$-surjective map $f: M_{1} \rightarrow$ $M_{2}$, then $f$ is of non-zero degree.

Proof We may assume that the map $f$ has been deformed to be a fiber-preserving map.

(a) Special Case. We first prove that when both $M_{1}$ and $M_{2}$ are circle bundles, and $f$ : $M_{1} \rightarrow M_{2}$ is $\pi_{1}$-surjective or of $\pi_{1}$-finite-index, $f$ is of non-zero degree.

If $f$ is of $\pi_{1}$-finite index, then we have a $\pi$-surjective lift $\tilde{f}: M_{1} \rightarrow \tilde{M}_{2}$ of $f$. By the rank consideration, the genus of the orbifold of $\tilde{M}_{2}$ must be also $g$. So below we assume that $f$ is $\pi_{1}$-surjective. 
Let $t_{1}$ and $t_{2}$ be circle fibers of $M_{1}$ and $M_{2}$, respectively. The restriction $f \mid$ on the fiber $t_{1}$ is not null-homotopy. Otherwise we have an epimorphism $\phi=\hat{f}_{*} \circ p: \pi_{1}(F)=\pi_{1}\left(M_{1}\right) /\left\langle t_{1}\right\rangle \rightarrow$ $\pi_{1}\left(M_{2}\right) \rightarrow \pi_{1}\left(M_{2}\right) /\left\langle t_{2}\right\rangle \pi_{1}(F)$, where $\hat{f}: F \rightarrow M_{2}$ is the induced map. It follows that the genus of $F_{1}$ is at least the genus of $F_{2}$. By the Hopfian property of surface group, $\phi$ must be an isomorphism. It follows that $\bar{f}_{*}: \pi_{1}(F) \rightarrow \pi_{1}\left(M_{2}\right)$ is an isomorphism. This is impossible since $M_{2}$ is a closed 3-manifold. It follows that indeed $f \mid: t_{1} \rightarrow t_{2}$ must be of degree 1 . Then $f: M_{1} \rightarrow M_{2}$ induces a map $\bar{f}: F \rightarrow F$ which is also $\pi_{1}$-surjective. $\bar{f}$ must be of degree 1 . A conclusion is that $f$ itself must be of degree 1 .

(b) General Case. We equip each regular fiber with the structure of a unit circle in the complex plane and the length of each singular fiber of type $(\alpha, \beta)$ is $\frac{2 \pi}{\alpha}$. Furthermore, we assume that the map $f$ has been deformed so that the restriction $f \mid: C_{1} \rightarrow C_{2}$ on each regular fiber is given by $e^{i \zeta} \mapsto e^{i k \zeta}$, where the constant $k$ is the mapping degree on regular fibers, and on the regular neighborhood of singular fiber is the "linear extension". Suppose the singular fibers of $M_{1}$ are of type $\left(\alpha_{1}, \beta_{1} ; \ldots ; \alpha_{m}, \beta_{m}\right)$ and the singular fibers of $M_{2}$ are of type $\left(\alpha_{1}^{\prime}, \beta_{1}^{\prime} ; \ldots ; \alpha_{n}^{\prime}, \beta_{n}^{\prime}\right)$. Let $l=\prod \alpha_{i} \alpha_{j}^{\prime}$. Let $\bar{M}_{i}=M_{i} / \mathbb{Z}_{l}$, where the cyclic group $\mathbb{Z}_{l}$ acts on $M_{i}$ such that (1) $\mathbb{Z}_{l}$ keeps each fiber invariant, (2) on each regular fiber, the action is given by $e^{i \zeta} \mapsto e^{i \zeta+\frac{2 \pi}{l}}$. Then one can verify (as we did in Example 3) that (1) both $\bar{M}_{1}$ and $\bar{M}_{2}$ are circle bundles over $F$, and (2) $f: M \rightarrow N$ induces a fiber-preserving map, $\bar{f}: \bar{M} \rightarrow \bar{N}$, and the following diagram is commutative:

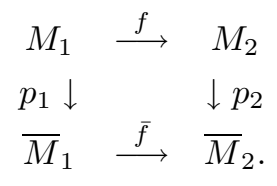

Since both $p_{1}, p_{2}$ and $f$ are of $\pi_{1}$-finite index, $\bar{f}$ is of $\pi$-finite index. By the special case we considered, $\bar{f}$ is of non-zero degree. So $f$ is of non-zero degree.

\section{$3 \quad$ On Epimorphisms Between 3-Manifold Groups}

In this section we study the following questions:

Question 3.1 Let $M_{i}$ be closed orientable aspherical 3-manifolds. Suppose there is an nontrivial epimorphism $\phi: \pi_{1}\left(M_{1}\right) \rightarrow \pi_{1}\left(M_{0}\right)$.

(A) Is rank $\pi_{1}\left(M_{1}\right)>\operatorname{rank} \pi_{1}\left(M_{0}\right)$ ?

(B) Is the Heegaard genus of $M_{1} \geq$ Heegaard genus $M_{0}$ ?

Moreover, given $M_{0}$ :

(C1) Is there a number $N_{M}$ such that any sequence of epimorphisms $\pi_{1}\left(M_{0}\right) \rightarrow \pi_{1}\left(M_{1}\right) \rightarrow$ $\ldots \rightarrow \pi_{1}\left(M_{n}\right)$ with $n \geq N_{M}$ contains an isomorphism?

(C2) Does any infinite sequence of epimorphisms $\pi_{1}\left(M_{0}\right) \rightarrow \pi_{1}\left(M_{1}\right) \rightarrow \ldots \rightarrow \pi_{1}\left(M_{n}\right) \rightarrow \ldots$ contain an isomorphism?

(D) Are there only finitely many $M_{i}$ with the same first Betti number, or the same $\pi_{1}$-rank, as that of $M_{0}$, for which there is an epimorphism $\pi_{1}\left(M_{0}\right) \rightarrow \pi_{1}\left(M_{i}\right)$ ? 
We remark that a positive answer for (B) of Question 3.1 implies a positive solution to the Poincaré Conjecture. From Example 1.4 of the Introduction, the answer to (D) is negative if we remove the condition on the first Betti number or $\pi_{1}$-rank on (D) of Question 3.1.

We describe, first, some examples of non-trivial $\pi_{1}$-surjective maps between two 3 -manifolds of the same rank, which give a negative answer to (A) of Question 3.1. Clearly those examples are all of non-zero degrees.

Example 3.1. Let $M$ be a Seifert manifold of normal form $\left(0 ; 0 ; 6, b_{1} ; 5, b_{2} ; 7, b_{3}\right)$. Let $\mathbb{Z}_{2}$ be a cyclic group acting on $M$ such that it induces the identity on the base space and standard rotation on each regular fiber. Then one verifies that $M / \mathbb{Z}_{2}$ is a Seifert manifold of normal form $\left(0 ; 0 ; 3, b_{1} ; 5,2 b_{2} ; 7,2 b_{3}\right)$. Now

$$
\pi_{1}(M)=\left\langle s_{1}, s_{2}, s_{3}, h \mid\left[s_{j}, h\right], s_{1}^{6} h^{b_{1}}, s_{2}^{5} h^{b_{2}}, s_{3}^{7} h^{b_{3}}, s_{1} s_{2} s_{3}\right\rangle
$$

and

$$
\pi_{1}\left(M / \mathbb{Z}_{2}\right)=\left\langle t_{1}, t_{2}, t_{3}, h^{\prime} \mid\left[t_{j}, h^{\prime}\right], t_{1}^{3} h^{\prime b_{1}}, t_{2}^{5} h^{\prime 2 b_{2}}, t_{3}^{7} h^{\prime 2 b_{3}}, t_{1} t_{2} t_{3}\right\rangle
$$

The quotient map $p: M \rightarrow M / \mathbb{Z}_{2}$ is a branched covering of degree 2 and $p_{*}$ sends $s_{j} \mapsto t_{j}$ and $h \mapsto h^{\prime 2}$. Since $\left(2, b_{1}\right)=1, p_{*}$ is surjective. By [13] these manifolds have rank 2 .

Examples 3.2. We now give some examples of $\pi_{1}$-surjective non-zero degree maps between hyperbolic manifolds of the same $\pi_{1}$ ranks.

Let $M$ be a closed orientable 3-manifold and $k \subset M$ be any hyperbolic fibered knot. Suppose the fiber $F$ has genus $g$. Let $M_{n}$ be the $n$-fold cyclic branched cover of $M$ over the knot $k$. Then the rank of $\pi_{1}\left(M_{n}\right)$ is bounded by $2 g+1$ for all $n$ and $M_{n}$ is hyperbolic when $n$ is large. If $k \mid n$, then $M_{n} \rightarrow M_{k}$ is a branched cover, which is $\pi_{1}$-surjective. So there must be infinitely many $\pi_{1}$-surjective branched coverings $M_{n} \rightarrow M_{k}$ between hyperbolic 3-manifolds of the same ranks.

A well-studied case is when $M_{n}$ is the $n$-fold cyclic branched cover of the figure-eight knot. Then for $n \geq 3$ the fundamental groups are all 2-generator, in fact, they are the Fibonacci groups $F(2,2 n)$ (see [17] for example), which are all hyperbolic if $n \geq 4$. By abelianizing $F(2,2 n)$ we see that all $M_{n}$ have their first Betti number zero (see [17] for example).

Example $3.3 \quad(1)$ Let $M(n, k)=\left(0 ; 0 ; 2^{k} 3, b_{1} ; 5,2^{n-k} b_{2} ; 7,2^{n-k} b_{3}\right)$. Similarly to Example 3.1 , we have a sequence of degree 2 branched coverings $M(n, n) \rightarrow \ldots \rightarrow M(n, 1) \rightarrow M(n, 0)$ of length $n+1$, which induces a sequence of epimorphisms of groups $\pi_{1}(M(n, n)) \rightarrow \ldots \rightarrow$ $\pi_{1}(M(n, 1)) \rightarrow \pi_{1}(M(n, 0))$ of rank 2 . Let $M$ be $\Sigma_{2} \times S^{1}$. Clearly $\pi_{1}(M)$ surjects onto $\mathbb{Z} * \mathbb{Z}$, then we have the sequence of epimorphisms

$$
\pi_{1}(M) \rightarrow \pi_{1}(M(n, n)) \rightarrow \ldots \rightarrow \pi_{1}(M(n, 1)) \rightarrow \pi_{1}(M(n, 0))
$$

of length $n+2$, where $n$ can be arbitrarily large.

Moreover, suppose we choose $b_{1}, b_{2}, b_{3}$ such that the Euler number of $M(n, n)$ is non-zero. Since each $M(N, k)$ has infinite $\pi_{1}$ and is the image of $M(n, n)$ under non-zero degree map, 
the Euler number of $M(n, k)$ is non-zero [18, Theorem 2]. It follows that $M(n, k)$ has neither a horizontal nor a vertical incompressible surface, and therefore all $M(n, k)$ are non-Haken [15].

(2) Let $M_{n}$ be the $n$-fold cyclic branched covering of $S^{3}$ over figure-eight knot as at the end of Example 3.2. Then we have a sequence of branched coverings of hyperbolic rational homology spheres $M_{4 k} \rightarrow \ldots \rightarrow M_{8} \rightarrow M_{4}$ of length $l$ which induces a sequence of epimorphisms of groups $\pi_{1}\left(M_{4 k}\right) \rightarrow \ldots \rightarrow \pi_{1}\left(M_{8}\right) \rightarrow \pi_{1}\left(M_{4}\right)$ with rank 2 . Let $M$ be a hyperbolic 3-manifold with $\pi_{1}(M)$ surjecting $\mathbb{Z} * \mathbb{Z}$ (as in Example 1.4). Then we have the sequence of epimorphisms

$$
\pi_{1}(M) \rightarrow \pi_{1}\left(M_{4 k}\right) \rightarrow \ldots \rightarrow \pi_{1}\left(M_{8}\right) \rightarrow \pi_{1}\left(M_{4}\right)
$$

of length $l+1$, where $l$ can be arbitrarily large.

The next result gives a partial positive answer to $(\mathrm{C} 2)$ of Question 3.1.

Theorem 3.4 Given $M_{0}$, and a sequence $M_{i}$ of closed orientable aspherical Seifert manifolds with epimorphisms $\pi_{1}\left(M_{0}\right) \rightarrow \pi_{1}\left(M_{1}\right) \rightarrow \ldots \rightarrow \pi_{1}\left(M_{n}\right) \rightarrow \ldots$, this sequence contains an isomorphism.

Proof By passing to an infinite subsequence, we may assume that all groups in the sequence have the same rank (each epimorphism in the subsequence is the composition of epimorphisms involved). Then each epimorphism $\phi_{i}: \pi_{1}\left(M_{i}\right) \rightarrow \pi_{1}\left(M_{i+1}\right)$ in the sequence can be realized by a map $f_{i}: M_{i} \rightarrow M_{i+1}$ of non-zero degree by Theorem 2.1. Moreover, the Seifert fibrations of the $M_{i}$ 's can be arranged so that each $f_{i}$ is fiber-preserving. Let $O_{i}$ be the orbifold of $M_{i}$. Then $\chi\left(O_{i}\right) \leq 0$ and we have the induced sequence of epimorphisms

$$
\pi_{1}\left(O_{0}\right) \rightarrow \pi_{1}\left(O_{1}\right) \rightarrow \ldots \rightarrow \pi_{1}\left(O_{n}\right) \rightarrow \ldots
$$

of Fuchsian groups. We therefore have a decreasing sequence

$$
-\chi\left(M_{0}\right) \geq-\chi\left(M_{1}\right) \geq \ldots-\chi\left(M_{n}\right) \geq \ldots
$$

The $\{-\chi(O)\}$ forms a well-ordered subset of reals, where $O$ runs over compact 2-orbifolds, $\chi\left(O_{k}\right)=\chi\left(O_{k+1}\right)$ for $k$ larger than a given $N$ (see [6, Lemmas 2.5 and 2.6] for details). Since there are at most finitely many orbifolds $O$ with given $\chi$, by passing to a subsequence, we may assume that all $O_{i}$ are the same.

Let $O_{i}=\left(g ; \alpha_{1}, \ldots, \alpha_{n}\right)$. Then $M_{i}=\left(g ; b_{i} ; \alpha_{1}, \beta_{1, i} ; \ldots ; \alpha_{n,}, \beta_{n, i}\right)$.

Since $0<\beta_{l, i}<\alpha_{l}$ for $l=1, \ldots n$, by passing to a further subsequence, we may assume that $\beta_{l, i}=\beta_{l}$, and finally we get $M_{i}=\left(g ; b_{i} ; \alpha_{1}, \beta_{1} ; \ldots ; \alpha_{n}, \beta_{n}\right)$. Moreover we may assume that all $b_{i} \neq 0$. Note that by [3, p. 680], all $M_{i}$ have the same first Betti number and the torsion part of $H_{1}\left(M_{i}, \mathbb{Z}\right)$ is unbounded if $b_{i}$ unbounded. Since epimorphisms on $\pi_{1}$ induce epimorphisms on the first homology groups, it follows that $b_{i}$ 's are bounded. Now we have $b_{i}=b_{j}$ for some $i, j$, then $M_{i}=M_{j}$ and by the Hopfian property of Seifert manifold groups, the epimorphism $\pi_{1}\left(M_{i}\right) \rightarrow \pi_{1}\left(M_{j}\right)$ is an isomorphism. Then in the sequence above there must be an isomorphism. Theorem 3.4 follows. 
We have seen that Theorem 2.1 plays an important role in the proof of Theorem 3.4. If the answer to Question 1.5 is also yes for hyperbolic 3-manifolds, this will lead to a positive answer to (C2) and (D) for hyperbolic 3-manifolds.

Proposition 3.5 Suppose Question 1.5 has a positive answer for hyperbolic 3-manifolds. Then for a given closed orientable hyperbolic 3-manifold $M_{0}$ :

(1) Any infinite sequence of epimorphisms $\pi_{1}\left(M_{0}\right) \rightarrow \pi_{1}\left(M_{1}\right) \rightarrow \ldots . \rightarrow \pi_{1}\left(M_{n}\right) \rightarrow \ldots$ contains an isomorphism, where all $M_{i}$ are closed orientable hyperbolic 3-manifolds.

(2) There are only finitely many closed orientable hyperbolic 3-manifolds $M_{i}$ with the same $\pi_{1}$-rank as that of $M_{0}$, for which there is an epimorphism $\pi_{1}\left(M_{0}\right) \rightarrow \pi_{1}\left(M_{i}\right)$.

Proof (1) By passing to an infinite subsequence we may assume all $\pi_{1}\left(M_{i}\right)$ have the same rank. Since we assume that Question 1.5 has a positive answer for hyperbolic 3-manifolds, this sequence is realized by a sequence of non-zero degree maps $M_{0} \rightarrow M_{1} \rightarrow \ldots \rightarrow M_{n} \rightarrow \ldots$

The rest of the proof is now standard. Since all maps $f_{i}: M_{i} \rightarrow M_{i+1}$ in the sequence are of non-zero degree, by Gromov's Theorem [19, Chapter 6], $v\left(M_{i}\right) \geq v\left(M_{i+1}\right)$, where $v\left(M_{i}\right)$ is the hyperbolic volume of $M_{i}$. By Thurston-Jøgenson's Theorem [19, Chapter 6], $v\left(M_{k}\right)$ must be a constant when $k$ is larger than a given integer $N$. Then by Gromov-Thurston's Theorem [19, Chapter 6], $f_{k}$ is homotopic to a homeomorphism, $k>N$, so $f_{k *}$ is an isomorphism.

For (2), since we again assume that Question of 1.5 has a positive answer for hyperbolic 3-manifolds, each $\phi_{i}: \pi_{1}\left(M_{0}\right) \rightarrow \pi_{i}\left(M_{i}\right)$ can be realized by a map of non-zero degree. By Soma's theorem [20], there are only finitely many such $M_{i}$.

Remark Without the assumption that Question 1.5 has positive answer for hyperbolic 3manifold, Proposition 3.5 (1) has been proved in [21]. Indeed, after discussing with D. Cooper, we tend to believe that Question 1.5 should have a negative answer for hyperbolic 3-manifolds, but we do not have complete argument yet.

We also note the following partial positive answer to (D) of Question 3.1 follows easily from the methods of Reid and Wang [4]:

Theorem 3.6 Suppose $M$ is a non-Haken hyperbolic 3-manifold. Then there are only finitely many closed orientable hyperbolic 3-manifolds $M_{i}$ for which there is an epimorphism $\pi_{1}(M) \rightarrow$ $\pi_{1}\left(M_{i}\right)$.

Proof As in [4] we use the character variety of $M$ to control possible target groups. Briefly if there are an infinite number of $M_{i}$ as in the statement of the theorem, we can produce a curve of characters $C$ in the character variety of $M$. By degenerating to a point at infinity of $C$ we getting a splitting of the group and hence an embedded incompressible surface in $M$ (see [22] and [4] for more on this). This is a contradiction.

\section{A Minimal Haken Manifold}

Let $E$ be the exterior of a trefoil knot with $m$ the meridian and $l$ the longitude. $E$ has a unique 
Seifert fibration with two singular fibers of indices 2 and 3, over the disc. Via this Seifert structure, we have a presentation

$$
\pi_{1}(E)=\left\langle a, b, c, t \mid a^{2} t, b^{3} t, a b c\right\rangle
$$

where $t$ is the regular Seifert fiber. Let $E_{1}$ and $E_{2}$ be homeomorphic to $E$ with meridian and longitudes $\left(m_{i}, l_{i}\right), i=1,2$. Now glue $E_{1}$ to $E_{2}$ via a homeomorphism $h: \partial E_{1} \rightarrow \partial E_{2}$ such that $h\left(l_{1}\right)=m_{2}$ and $h\left(m_{1}\right)=l_{2}^{-1}$. Let $M$ denote the resulting manifold, which is a closed graph manifold. The main theorem of this section is:

Theorem 4.1 $\quad M$ is a minimal closed Haken 3-manifold among all 3-manifolds satisfying Thurston's geometric conjecture.

We begin the proof by collecting some elementary facts.

Lemma 4.2 (1) For any representation $\phi: \pi_{1}(E) \rightarrow \mathrm{SL}(2, \mathbb{C})$, if $\phi(t) \neq 1$, then the image $\phi\left(\pi_{1}(E)\right)$ is a cyclic group $\langle\lambda\rangle$. Moreover, we must have $\phi(a)=\lambda^{-2}, \phi(b)=\lambda^{-3}, \phi(c)=\lambda^{5}$, and $\phi(t)=\lambda^{6}$.

(2) In $\pi_{1}(T)$, where $T=\partial E$, we have $m=t c^{-1}$ and $l=t^{-5} c^{6}$. (Equivalently, $t=6 m+l$ and $c=5 m+l$.) Hence $h\left(t_{1}^{-5} c_{1}^{6}\right)=t_{2} c_{2}^{-1}$ and $h\left(t_{1} c_{1}^{-1}\right)=t_{2}^{5} c_{2}^{-6}$.

(3) $M$ is an integral homology 3-sphere.

(4) The only 2-sided incompressible surface is the incompressible torus $T$, which separates $M$ into $E_{1}$ and $E_{2}$.

Proof The main part of (1) follows from [23, Prop. 3] and the fact that $H_{1}(E, \mathbb{Z})$ is cyclic. (2) and (3) and the remaining parts of (1) are just direct calculations. Finally, to establish (4) we observe the following. Since the trefoil knot is 2-bridge $E$ cannot contain a closed embedded essential surface by [24]. If $M$ contained an embedded incompressible surface $\neq T$, it would follow from the remark above and the gluing homeomorphism that $E$ would have a boundary slope 1/0. However, [22, Theorem 2.0.3] then implies the existence of a closed embedded essential surface in $E$.

To show that $M$ is minimal, we assume to the contrary that there is a degree one map $f: M \rightarrow N$, where $N$ is irreducible, $N \neq M$, and $N \neq S^{3}$. First, since $M$ is a graph manifold, its Gromov norm is zero, so $N$ cannot be a hyperbolic 3-manifold by [Theorem, Chapter 6]. Moreover, it is well-known that $N$ must be an integer homology sphere, [4, Lemma 3.1]. The proof of Theorem 4.1 will be finished by Lemmas $4.3,4.4$ and 4.5 below.

Lemma 4.3 $N$ is non-Haken.

Proof Suppose $N$ is Haken, and let $F \subset N$ be an embedded incompressible surface. We may deform $f$ so that $f^{-1}(F)$ is an incompressible surface in $M$. By (4) of Lemma $4.2, f^{-1}(F)$ must consist of parallel copies of $T$. By standard 3-manifold topology, we can further deform $f$ so that $f^{-1}(F)=T$. It follows that $F$ is a 2 -sphere or torus. Since $N$ is irreducible, $F$ must be a torus separating $N$ into two parts $N_{1}$ and $N_{2}$. Furthermore, the map $f$ can be decomposed into two proper degree-one maps $f \mid: E_{i} \rightarrow N_{i}$. However, $E_{i}$ is a minimal 3-manifold among knot 
complements in 3-manifolds via proper degree-one maps [1]. Thus, each $f \mid$ is a homeomorphism, and it follows that $f$ itself is homotopic to a homeomorphism.

Lemma 4.4 $\quad N$ is not a Seifert manifold with finite fundamental group (other than possibly $\left.S^{3}\right)$.

Proof By (3) of Lemma 4.2, if $N$ is a Seifert fibered manifold of finite fundamental group and $N \neq S^{3}$, it must be the Poincaré Homology 3 -sphere $P$. Note $\pi_{1}(P)$ surjects onto $A_{5}$, the alternating group on 5 letters. In particular, (as is well known) $A_{5}$ is a subgroup of PSL $(2, \mathbb{C})$ since $\mathrm{SO}(3)$ can be identified with $\operatorname{PSU}(2)$, and the latter is a subgroup of $\operatorname{PSL}(2, \mathbb{C})$. To prove the lemma, it suffices to prove that the image group of any representation of $\phi: \pi_{1}(M) \mapsto$ $\operatorname{PSL}(2, \mathbb{C})$ cannot be $A_{5}$.

Case (1) If $\phi\left(t_{1}\right) \neq 1$ and $\phi\left(t_{2}\right) \neq 1$, by (1) of Lemma 4.2, the whole image $\phi\left(\pi_{1}(M)\right)$ must be a cyclic group (actually trivial).

Case (2) Without loss of generality, we may assume that $\phi\left(t_{1}\right)=1$ and $\phi\left(t_{2}\right) \neq 1$. By (1) and (2) of Lemma 4.2, $\phi: \pi_{1}(M) \rightarrow \operatorname{PSL}(2, \mathbb{C})$ factors as $\nu: \pi_{1}(M) \rightarrow G$ and $\mu: G \rightarrow$ $\operatorname{PSL}(2, \mathbb{C})$, where $G$ is generated by two groups described in (a) and (b) below:

(a) $\nu\left(\pi_{1}\left(E_{1}\right)\right)=\left\langle a_{1}, b_{1}, c_{1} \mid a_{1}^{2}, b_{1}^{3}, a_{1} b_{1} c_{1}\right\rangle$, (b) A cyclic group $\left\langle\lambda_{2}\right\rangle$ such that $\nu\left(c_{2}\right)=\lambda_{2}^{5}$, $\nu\left(t_{2}\right)=\lambda_{2}^{6}$.

Since $h\left(t_{1} c_{1}^{-1}\right)=t_{2}^{5} c_{2}^{-6}$, we have $\nu\left(h\left(c_{1}^{-1}\right)\right)=\nu\left(h\left(t_{1} c_{1}^{-1}\right)\right)=\nu\left(t_{2}^{5} c_{2}^{-6}\right)=1$. It follows that

$$
G=\left\langle a_{1}, b_{1}, c_{1} \mid a_{1}^{2}, b_{1}^{3}, a_{1} b_{1} c_{1}, c_{1}\right\rangle=\left\langle a_{1}, b_{1} \mid a_{1}^{2}, b_{1}^{3}, a_{1} b_{1}\right\rangle,
$$

which is the trivial group.

Case (3) $\quad \phi\left(t_{1}\right)=1$ and $\phi\left(t_{2}\right)=1$. In this case $\phi: \pi_{1}(M) \rightarrow \operatorname{PSL}(2, \mathbb{C})$ factors through a group $G$ via a map $\nu: \pi_{1}(M) \rightarrow G$, with $\nu\left(\pi_{1}\left(E_{i}\right)\right)$ being the quotient of $G_{i}=\left\langle a_{i}, b_{i}, c_{i}\right| a_{i}^{2}$, $\left.b_{i}^{3}, a_{i} b_{i} c_{i}\right\rangle, i=1,2$. Moreover, by (2) of Lemma 4.2 we have that in the quotient $c_{1}=c_{2}^{6}$ and $c_{2}=c_{1}^{-6}$. Immediately we have that $c_{1}^{37}=1$ and $c_{2}^{37}=1$ and finally

$$
G=\left\langle a_{i}, b_{i}, c_{i}, i=1,2 \mid a_{i}^{2}, b_{i}^{3}, a_{i} b_{i} c_{i}, c_{i}^{37}, c_{1}=c_{2}^{6}, i=1,2\right\rangle .
$$

Suppose there is a homomorphism $\mu: G \rightarrow A_{5}$. Since the order of $c_{i}$ is 37 , and $A_{5}$ has order 60 , under the homomorphism $\mu$ the images of $c_{1}$ and $c_{2}$ must be trivial. It follows that $\mu: G \rightarrow A_{5}$ can factor through the group $G^{\prime}$,

$$
G^{\prime}=\left\langle a_{1}, b_{1}, \mid a_{1}^{2}, b_{1}^{3}, a_{1} b_{1}\right\rangle *\left\langle a_{2}, b_{2}, \mid a_{2}^{2}, b_{2}^{3}, a_{2} b_{2}\right\rangle,
$$

but as said above, this is trivial.

Lemma 4.5 $N$ is not a Seifert manifold with infinite $\pi_{1}$.

The proof of Lemma 4.5 requires a sequence of additional lemmas. We suppose below that $N$ is a Seifert manifold with infinite $\pi_{1}$. By Lemma 4.3, we may assume that $N$ is non-Haken. Hence $N$ must be a Seifert manifold with three singular fibers over $S^{2}$.

We begin by establishing: 
Lemma 4.5.1 (1) Suppose $\Delta \subset \operatorname{PSL}(2, \mathbb{R})$ is a triangle group and $\phi: \pi_{1}(2,3, l) \rightarrow \Delta$ is of finite index. Then the image of $\phi$ is a hyperbolic triangle group isomorphic to $\pi_{1}(2,3, k)$, where $k \mid l$.

(2) Suppose a Seifert manifold $N$ is an integer homology 3-sphere with infinite $\pi_{1}$ and orbifold $O=\left(a_{1}, a_{2}, a_{3}\right)$. Then $\operatorname{gcd}\left(a_{i}, a_{j}\right)=1$ for $i, j=1,2,3$, and $O$ is a hyperbolic orbifold.

Proof (1) Let $x^{\prime}, y^{\prime}$ be the order 2 and order 3 elements which generate $\pi_{1}(2,3, l)$ such that $x^{\prime} y^{\prime}$ is of order $l$. Use $x$ and $y$ to denote their images in $\operatorname{PSL}(2, \mathbb{R})$, then $x$ and $y$ generate the image of $\phi$. Since the image of $\phi$ is of finite index in $\Delta$, it must be co-compact and of rank 2 . By the well-known facts, the image is a triangle group with $x^{2}=y^{3}=(x y)^{k}=1$, where $k \mid l$.

(2) follows from $[3$, p. $680(d)]$.

Lemma 4.5.2 There is a simple closed curve in the kernel of $f \mid: T \rightarrow N$.

Proof Since $\pi_{1}(N)$ is torsion free and $T$ is a torus, to prove the lemma, we need only to show that the kernel of $f \mid: T \rightarrow N$ is non-trivial.

Suppose $f\left(t_{1}\right) \neq 1$; otherwise, the claim is proved. Note that all elements in $f\left(\pi_{1}\left(E_{1}\right)\right)$ commute with $f\left(t_{1}\right)$. If $f\left(t_{1}\right)$ is not the fiber $t$ of $N$, then either

$$
f\left(\pi_{1}\left(E_{1}\right)\right)=f\left(t_{1}\right) \text { or } f\left(\pi_{1}\left(E_{1}\right)\right)=\left\langle f\left(t_{1}\right), f\left(c_{1}\right)\right\rangle=\mathbb{Z} \oplus \mathbb{Z} .
$$

The second case is not possible since $H_{1}\left(E_{1} ; \mathbb{Z}\right)=\mathbb{Z}$. In the first case we deduce that $\operatorname{ker}\left(\left.f\right|_{T}\right)_{*}$ is non-trivial. Similarly if $f\left(t_{2}\right)$ is not the fiber $t$ of $N$, then $\operatorname{ker}\left(\left.f\right|_{T}\right)_{*}$ is non-trivial. Suppose $f\left(t_{1}\right)=t=f\left(t_{2}\right)$. Since $t_{1}$ and $t_{2}$ do not coincide up to an isotopy, still we have that $\operatorname{ker}\left(\left.f\right|_{T}\right)_{*}$ is non-trivial.

Let $C$ be the simple closed curve provided by Lemma 4.5.2. Suppose $C=p m_{1}+q l_{1}$ on $\partial E_{1}$. Then $C=-q m_{2}+p l_{2}$. By (1) of Lemma 4.2 we have $p m+g l=(p-5 q) t+(-p+6 q) c$ and $-q m+p l=(-q-5 p) t+(q+6 p) c$. So the degree 1 map $f$ factors through $f: M \rightarrow$ $N_{1} \cup_{S^{1}} N_{2} \rightarrow N$, where $N_{1}$ and $E_{2}^{\prime}$ are Seifert manifolds whose normal forms are given by $(2,1 ; 3,1 ;-p+6 q, p-5 q)$ and $(2,1 ; 3,1 ; q+6 p,-5 p-q)$, respectively, and the two cores of the surgery solid tori are identified. If $\left.f\right|_{*}\left(\pi_{1}\left(N_{1}\right)\right) \neq \pi_{1}(N)$ and $\left.f\right|_{*}\left(\pi_{1}\left(N_{2}\right)\right) \neq \pi_{1}(N)$ then $\pi_{1}(N)$ can be presented as a non-trivial free product with amalgamation by the classical result (see [22] for example). It follows that $N$ will be Haken, contrary to Lemma 4.3. Thus without loss of generality, we assume that $\left.f\right|_{*}\left(\pi_{1}\left(N_{1}\right)\right)=\pi_{1}(N)$.

Lemma 4.5.3 $\left.f\right|_{N_{2}}$ is of non-zero degree.

Proof Let $\tilde{E}$ be the covering of $N$ corresponding to $\left.f\right|_{*}\left(\pi_{1}\left(N_{2}\right)\right)$. Then $f: N_{2} \rightarrow N$ lifts to $\tilde{f}: N_{2} \rightarrow \tilde{E}$, which is $\pi_{1}$-surjective. If $\left.f\right|_{*}\left(\pi_{1}\left(N_{2}\right)\right) \subset \pi_{1}(N)$ is of finite index, then $\tilde{E}$ is a closed Seifert manifold. Since both $\pi_{1}\left(N_{1}\right)$ and $\pi_{1}(N)$ are of rank $2, \pi_{1}(\tilde{E})$ must also be of rank 2 . Then $\tilde{f}$ is of non-zero degree by Theorem 2.1. Hence $\left.f\right|_{N_{2}}$ is of non-zero degree.

Below we show $\left.f\right|_{*}\left(\pi_{1}\left(N_{2}\right)\right) \subset \pi_{1}(N)$ must be of finite index. Otherwise $\tilde{E}$ is a non-compact, aspherical Seifert manifold, for which it is known that either the rank of $H_{1}(\tilde{E})$ is positive or $\pi_{1}(\tilde{E})$ is trivial. Since $\left.f\right|_{*}\left(\pi_{1}\left(N_{2}\right)\right)$ is not trivial and $N_{2}$ is a rational homology sphere, all of the above cases are ruled out. So $\left.f\right|_{*}\left(\pi_{1}\left(N_{2}\right)\right)$ must be of finite index in $\pi_{1}(N)$. 
Since $N_{1}$ and $N_{2}$ are in symmetry position, we have both $f \mid N_{1}$ and $f \mid N_{2}$ are of non-zero degree.

By Lemma 4.5.3, we may assume that $f \mid N_{i}$ is fiber preserving. Then $f \mid N_{i}$ induces an homomorphism $\phi_{i}: \pi_{1}\left(O_{i}\right) \rightarrow \pi_{1}(O)$; in particular, $\phi_{1}$ is surjective and $\phi_{2}$ is of finite index, where $O_{1}=(2,3,6 q-p), O_{2}=(2,3,6 q+p)$ and $O=\left(a_{1}, a_{2}, a_{3}\right)$ are orbifolds of $N_{1}, N_{2}$ and $N$, respectively. Since $N$ is an integral homology 3 -sphere of infinite $\pi_{1}$, it follows that $\pi_{1}(O)$ is isomorphic to a hyperbolic triangle group. Since $\phi_{1}: G_{1} \rightarrow G$ is surjective, it follows that $O=(2,3, k)$, where $k \mid 6 q-p$ by Lemma 4.5.1(1). Since $\phi_{2}$ is of finite index, the image of $\phi_{2}$ is a hyperbolic triangle group $\pi_{1}\left(2,3, k^{\prime}\right)$ with $k^{\prime} \mid 6 q+p$ by Lemma 4.5.1(1); moreover, $k^{\prime} \mid k$. It is easy to see that $k^{\prime}$ is a divisor of both $12 q$ and $2 p$. Since $p$ and $q$ are coprime, the great common divisor of $12 q$ and $2 p$ is 12 . So $k^{\prime}$ is either 2 , or 3 , or 4 , or 6 , or 12 . Then $N$ can not be an integer homology sphere by Lemma 4.5.1(2).

\section{References}

[1] Boileau M., Wang S. C., Non-zero degree maps and surface bundles over $S^{1}$, J. Diff. Geom., 1996, 43: 789-908.

[2] Callahan P. J., Reid A. W., Hyperbolic structures on knot complements, Chaos, Solitons and Fractals, 1998, 9: 705-738.

[3] Hayat-Legrand C., Wang S. C., Zieschang H., Minimal Seifert manifolds, Math. Annalen., 1997, 308: 673-700.

[4] Reid A. W., Wang S. C., Non-Haken 3-manifolds are not large with respect to mappings of non-zero degree, Comm. Analysis \& Geom, 1999, 7(1): 105-132.

[5] Kirby R., Problems in Low Dimensional Topology, Geometric Topology, Edited by Kazez H., AMS/IP 1997.

[6] Rong Y., Degree one maps between geometric 3-manifolds, Trans. A. M. S., 1992, 332: 411-436.

[7] Soma T., Sequences of degree-one maps between geometric 3-manifolds, Math. Annalen., 2000, 316: 733742.

[8] Soma T., Maps of non-zero degree to hyperbolic 3-manifolds, J. Diff. Geom., 1998, 49: 517-546.

[9] Hayat-Legrand C., Wang S. C., Zieschang H., Any 3-manifold 1-dominates at most finitely many 3-manifolds supporting $S^{3}$ geometry, Proc. AMS, to appear.

[10] Wang S. C., Zhou Q., Any 3-manifold 1-dominates at most finitely many geometric 3-manifolds, Math. Annalen, to appear.

[11] Rong Y., Maps between Seifert fibered spaces of infinite $\pi_{1}$, Pacific J. Math., (1993), 160: 143-154.

[12] Zieschang H., Vogt E., Coldeway H., Surfaces and Planar Discontinuous Group, Lecture Notes in Math. 835, Berlin: Springer-Verlag 1980.

[13] Boileau M., Zieschang H., Heegaard genus of closed orientable Seifert 3-manifolds, Invent. Math., 1984, 76: 455-468.

[14] Hass J., Minimal surfaces in manifolds with $S^{1}$ actions and the simple loop conjecture for Seifert fiber spaces, Proc. AMS, 1987 99: 383-388.

[15] Jaco W., Topology of Three Manifolds, Regional Conference Series in Mathematics, 43, AMS Providence, RI 1980.

[16] Huang C., On maps between Seifert manifolds, Acta Scien. Nature Peking Univ., 1999, 35: 577-588.

[17] Maclachlan C., Reid A. W., Generalised Fibonacci manifolds, Transformation Groups, 1997, 2: 165-182.

[18] Wang S. C., The existence of maps of non-zero degree between aspherical 3-manifolds, Math. Zeit., 1991, 208: $147-160$.

[19] Thurston W. P., The Geometry and Topology of 3-manifolds, Princeton University, Mimeographed Notes, 1979.

[20] Scott G. P., The geometries of 3-manifolds, Bull. London Math. Soc., 1983, 15: 401-487.

[21] Soma T., Epimorphisms sequences between hyperbolic 3-manifold groups, Proc. AMS, to appear.

[22] Culler M., Gordon C. McA., Luecke J., Shalen P. B., Dehn surgery on knots, Annals of Math., 1987 125: 237-300.

[23] Motegi K., Haken manifolds and representations of their fundamental groups in $\mathrm{SL}(2, \mathbb{C})$, Topology and Its Applications, 1988, 29: 207-212.

[24] Hatcher A., Thurston W., Incompressible surfaces in 2-bridge knot complements, Invent. Math., 1985, 79: $225-246$. 